\title{
Editorial
}

\section{Post-Traumatic Stress Disorder and the Care of Persons Living With HIV/AIDS}

\author{
Steven S. Coughlin ${ }^{*}, 1,2, \S$
}

\author{
${ }^{I}$ Epidemiology Program, Post Deployment Health, Office of Public Health, Department of Veterans Affairs, \\ Washington, DC, USA \\ ${ }^{2}$ Department of Epidemiology, Rollins School of Public Health, Emory University, Atlanta, GA, USA
}

In conjunction with medical and scientific advances that have improved the survival and quality of life of persons living with HIV/AIDS, there has been increasing interest in examining post-traumatic stress disorder (PTSD) in HIV positive persons. Studies of comorbid HIV/AIDS and PTSD have focused on a wide variety of demographic, cultural, and socioeconomic subgroups of diverse populations including women [1-3], socioeconomically disadvantaged persons [4, 5], gay men [6], and adolescents and young adults in developed countries such as the U.S. and Great Britain [7]. Other studies have focused on adults or children in African countries impacted by the AIDS pandemic $[8,9]$. In the U.S., PTSD rates have been reported to be $10.4 \%$ to $42.2 \%$ in treatment seeking and convenience samples of patients with HIV/AIDS $[2,10]$. Several factors may account for the cooccurrence of HIV/AIDS and PTSD and other anxiety and mood disorders such as major depression. These include the potentially traumatic nature of being diagnosed with a diagnosis that is often perceived as life-threatening; the relatively high rates of traumatic exposures (physical assault, sexual trauma, or the sudden, unexpected death of a close family member or friend) that have been reported in studies of persons with HIV/AIDS; and patient perceptions of AIDS-related stigma [2, 11].

Current medical and public health recommendations in the U.S., such as routine voluntary screening for HIV infection as a normal part of medical practice and HIV counseling, testing, and referral for persons at high risk for HIV who receive HIV testing in nonclinical settings such as community-based organizations, and similar recommendations in other countries, help to combat social stigma and to increase the numbers of patients who receive appropriate and potentially life-saving therapy [12]. Although PTSD may develop following an HIV/AIDS diagnosis, the reverse is also true as some persons suffering from PTSD, substance abuse, or other psychiatric illness are more likely to engage in risky behaviors including unsafe sex [13]. Early life stressors such as physical abuse, sexual abuse, neglect, verbal violence, or witnessed violence have been associated

*Address correspondence to this author at the Epidemiology Program, Post Deployment Health (10P3A), Office of Public Health, 810 Vermont Ave., NW, Washington, DC 20420, USA; Tel: (202) 266-4656; Fax: (202) 4955973; E-mail: steven.coughlin@va.gov

${ }^{\S}$ Editor-in-Chief with incident HIV infection in 13,274 U.S. men who participated in the National Epidemiologic Survey on Alcohol and Related Conditions, 2004-2005 [14]. Women, children, and sexual minorities may be exposed to HIV as a result of sexual trauma.

Results from studies conducted in the U.S., Great Britain, South Africa, and other countries indicate that the cooccurrence of HIV/AIDS and PTSD results in special challenges for people living with HIV/AIDS and their health care providers, and that it is important to consider the ages, gender, sexual orientation, socioeconomic background, and culture of at-risk persons. Studies have shown that many women living with HIV/AIDS have PTSD stemming from sexual or physical assault or other adverse life experiences. Katz and Nevid [2] examined risk factors for PTSD symptomatology in a sample of 102 HIV positive women who were recruited from HIV treatment centers in the New York City metropolitan area. Factors found to be associated with PTSD symptoms included greater number of HIVrelated physical symptoms, prior history of trauma, greater perception of stigma, and less social support. About $15 \%$ of the women in the study had probable PTSD and an additional $20 \%$ had subsyndromal PTSD based on PTSD Checklist scores [2], although the study was limited by the small sample size and possible selection bias. Young persons living with HIV/AIDS are another vulnerable group in society that is often subject to physical violence and other traumatic experiences. Radcliffe et al. [7] examined PTSD in a small sample of 30 adolescents and young adults living with HIV/AIDS, who were seen at an outpatient clinic in Philadelphia. About $47 \%$ of the survey respondents in this small, selected sample reported traumatic events such as physical assault, sexual abuse, or being abandoned by a caregiver. The majority of the study participants $(57 \%)$ reported PTSD symptomatology but the percentage who met full criteria for PTSD was lower. Sexual minorities including gay men are additional population subgroups that have been the focus of studies on PTSD and HIV/AIDS. For example, Theuninck et al. [6] examined the relationship between HIVrelated events (including receiving the diagnosis, treatment, physical symptoms, and witnessing HIV-related death) and PTSD symptoms in a self-selected sample of 100 gay men living with HIV who were residents of Great Britain. About $33 \%$ met criteria for PTSD, although the survey may have been biased by selection factors and the use of self-reported information. Receiving medical treatment, experiencing 
physical symptoms, and witnessing HIV-related death were associated with PTSD symptoms [6]. Martin and Kagee [8] examined the percentage of persons who met criteria for lifetime PTSD and HIV-related PTSD among 85 patients attending public health clinics in the Western Cape, South Africa who had been recently diagnosed with HIV. The PTSD module of the Composite International Diagnostic Interview (CIDI) was used in their study. The rate of lifetime PTSD and incidence of PTSD considered to be HIV-related was 54.1\% (95\% CI 43.6-64.3\%) and 40\% (95\% 30.2$50.6 \%$, respectively, although the generalizability of the findings is uncertain. In some studies of persons living with HIV/AIDS, the researchers have attempted to distinguish between PTSD related to an HIV/AIDS diagnosis from PTSD stemming from other traumatic experiences such as sexual assault [2, 6-8]. Taken overall, results from published studies indicate that several factors may increase vulnerability to PTSD or increase symptoms of the disorder among persons living with HIV/AIDS.

The distress associated with PTSD symptoms can interfere with daily functioning and make it more difficult for patients to sustain healthy behaviors such as physical activity, smoking cessation, avoidance of alcohol abuse, and safer sex practices $[13,15]$. Studies have shown that PTSD symptoms can make it more difficult for persons living with HIV/AIDS to adhere to life-saving therapy over time. Researchers have noted that, in some persons facing HIV/AIDSrelated life challenges, medication use may serve as a reminder of a traumatic diagnosis or treatment experience, and patients may avoid taking medications in order to avoid distressing thoughts about their illness or because of dissociation [16, 17]. In addition, patient distress may be increased when returning to the site of the original diagnosis or the location of medical care [7].

\section{ADHERENCE WITH TREATMENT RECOMMEN- DATIONS}

Although strict medication adherence is needed for highly active antiretroviral therapy (HAART) to be effective, studies have shown that some persons living with HIV/AIDS self report low levels of adherence [18, 19]. Findings from clinical studies have demonstrated that PTSD can have a negative impact on patient adherence with HAART. Both PTSD and depression have been associated with decreased adherence with HAART regimens and with the faster progression of HIV/AIDS [4, 16, 18, 20-22]. For example, Delahanty et al. [16] examined PTSD symptomatology and adherence with HAART in a sample of 110 persons living with HIV. The participants were recruited from an AIDS service organization in a Midwestern city in the U.S. The researchers found that having a detectable viral load was significantly related to patient reports of any missed doses in the past 2 days, past week, or past 3 months. In addition, PTSD symptoms were found to be associated with lower reports of adherence to HAART [16]. A further finding was that avoidance symptoms were related to missed medical appointments and taking AIDS medications off schedule. Reilly et al. [20] examined PTSD in a convenience sample of $145 \mathrm{HIV}$-infected patients who returned to care at an outpatient clinic in New Orleans following Hurricane Katrina. Those with PTSD were more likely to have had interruptions in their medication immediately following the disaster and were more likely to have detectable plasma viral loads and CD4 cell counts $<200 / \mathrm{mm}^{3}$ two years later [20]. Additional information about the medical adherence challenges faced by people living with HIV/AIDS was obtained by Saren et al. [23] as part of a 12-week outcome study of an Internet paging system to increase HIV medical adherence. The participants included 59 men and 16 women who reported at the time of the initial screening at a community health center in Boston that they typically took less than $90 \%$ of their prescribed medications for HIV. The researchers, who assessed PTSD symptoms using the Posttraumatic Diagnostic Scale, found that over one half of the sample met criteria for PTSD [23].

\section{FURTHER RESEARCH TO IMPROVE THE CARE OF PERSONS WITH COMORBID HIV/AIDS AND PTSD}

Additional studies are needed to examine the effects of PTSD on the progression of HIV in diverse populations [2]. Studies to date have often had important study design limitations such as the use of self-reported information, a crosssectional design, inadequate sample sizes, or the potential for selection bias. Although a sizeable body of research has examined the effectiveness of interventions to help people living with HIV/AIDS adhere to HAART regimens and manage their health through self-care opportunities, relatively few studies have specifically examined the challenges faced by people living with comorbid HIV/AIDS and PTSD. Studies that take into account patient-related factors such as age, gender, sexual orientation, socioeconomic background, and culture, and factors related to health care systems, are likely to be particularly informative. The age, gender, and socioeconomic status of HIV/AIDS patients affect not only their risk of having comorbid PTSD but also considerations for care such as strategies for optimizing treatment and quality of life. In many countries, HIV-positive persons live in environments characterized by poverty, violence, or other societal problems $[8,11,24]$. Additional scientific and medical information is also needed about gender differences in treatment adherence and exposure to trauma in women and men with comorbid HIV/AIDS and PTSD [1, 24]. Studies of PTSD among children and adolescents living with HIV/ AIDS are also important. The implications of PTSD for the care of persons living with HIV/AIDS extend well beyond treatment adherence to include such considerations as fostering a safe and supportive home and social environment. Future research should include longitudinal studies that add to current knowledge about the ways in which people suffering from HIV/AIDS and PTSD (and other important comorbid conditions such as major depression) navigate health care systems, manage self-care behaviors, and cope with the challenges of their illness and other life stressors over time [7,23].

\section{REFERENCES}

[1] Simoni JM, Ng MT. Trauma, coping, and depression among women with HIV/AIDS in New York City. AIDS Care 2000; 12: 567-80.

[2] Katz S, Nevid JS. Risk factors associated with posttraumatic stress disorder symptomatology in HIV-infected women. AIDS Patient Care STDS 2005; 19: 110-20.

[3] Beckerman NL, Auerbach C. PTSD and HIV in women: the role of gender in this dual diagnosis. Women Health 2011; 51: 497-510. 
[4] Simoni JM, Frick PA, Lockhart D, Liebovitz D. Mediators of social support and antiretroviral adherence among an indigent population in New York City. AIDS Patient Care STDS 2002; 16: 431-9.

[5] Soller M, Kharrazi N, Prentiss D, Cummings S, Balmas G, Koopman $\mathrm{C}$, et al. Utilization of psychiatric services among lowincome HIV-infected patients with psychiatric comorbidity. AIDS Care 2011 [Epub ahead of print].

[6] Theuninck AC, Lake N, Gibson S. HIV-related posttraumatic stress disorder: investigating the traumatic events. AIDS Patient Care STDS. 2010; 24: 485-91.

[7] Radcliffe J, Fleisher CL, Hawkins LA, et al. Posttraumatic stress and trauma history in adolescents and young adults with HIV. AIDS Patient Care STDS. 2007; 21: 501-8.

[8] Martin L, Kagee A. Lifetime and HIV-related PTSD among persons recently diagnosed with HIV. AIDS Behav 2011; 15: 12531.

[9] Cluver LD, Orkin M, Gardner F, Boyes ME. Persisting mental health problems among AIDS-orphaned children in South Africa. J Child Psychol Psychiatry 2011 [Epub ahead of print].

[10] Martinez A, Israelski D, Walker C, Koopman C. Post-traumatic stress disorder in women attending human immunodeficiency virus outpatient clinics. AIDS Patient Care STDS 2002; 16: 283-91.

[11] Brief DJ, Bollinger AR, Vielhauer MJ, et al. Understanding the interface of HIV, trauma, post-traumatic stress disorder, and substance use and its implications for health outcomes. AIDS Care 2004; 16 (Supplement 1): S97-S120.

[12] Centers for Disease Control and Prevention. Revised recommendations for HIV testing of adults, adolescents, and pregnant women in health-care settings. Morb Mortal Wkly Rep 2006; 55 (RR14): 1-17.

[13] Cavanaugh CE, Hansen NB, Sullivan TP. HIV sexual risk behavior among low-income women experiencing intimate partner violence: the role of posttraumatic stress disorder. AIDS Behav 2010; 14: 318-27.

[14] Reisner SL, Falb KL, Mimiaga MJ. Early life traumatic stressors and the mediating role of PTSD in incident HIV infection among US men, comparisons by sexual orientation and race/ethnicity: results from the NESARC, 2004-2005. J Acquir Immune Defic Syndr 2011; 57: 340-50.

[15] Schnurr PP, Green BL. Understanding relationships among trauma, post-traumatic stress disorder, and health outcomes. Adv Mind Body Med 2004; 20: 18-29.

[16] Delahanty DL, Bogart LM, Figler JL. Posttraumatic stress disorder symptoms, salivary cortisol, medication adherence, and CD4 levels in HIV-positive individuals. AIDS Care 2004; 16: 247-60.

[17] Keuroghlian AS, Kamen CS, Neri E, et al. Trauma, dissociation, and antiretroviral adherence among persons living with HIV/AIDS. J Psychiatr Res 2011; 45: 942-8.

[18] Sledjeski EM, Delahanty DL, Bogart LM. Incidence and impact of posttraumatic stress disorder and comorbid depression on adherence to HAART and CD4+ counts in people living with HIV. AIDS Patient Care STDS 2005; 19: 728-36.

[19] Chesney MA, Ickovics JR, Chambers DB, et al. Self-reported adherence to antiretroviral medications among participants in HIV clinical trials: the AACTG adherence instruments. Patient Care Committee \& Adherence Working Group of the Outcomes Committee of the Adult AIDS Clinical Trials Group (AACTG). AIDS Care 2000; 12: 255-66.

[20] Reilly KH, Clark RA, Schmidt N, et al. The effect of posttraumatic stress disorder on HIV disease progression following hurricane Katrina. AIDS Care 2009; 21: 1298-305.

[21] Cohen MA, Alfonso CA, Hoffman RG, et al. The impact of PTSD on treatment adherence in persons with HIV infection. Gen Hosp Psychiatry 2001; 23: 294-6.

[22] Schuman P, Ohmit SE, Cohen M, et al. Prescription of and adherence to antiretroviral therapy among women with AIDS. AIDS Behav 2001; 5: 371-8.

[23] Safren SA, Gershuny BS, Hendriksen E. Symptoms of posttraumatic stress and death anxiety in persons with HIV and medication adherence difficulties. AIDS Patient Care STDS 2003; 17: 657-64.

[24] Olley BO, Zeier MD, Seedat S, Stein DJ. Post-traumatic stress disorder among recently diagnosed patients with HIV/AIDS in South Africa. AIDS Care 2005; 17: 550-7. 